\title{
De l'imagerie quantique aux télécommunications : une histoire de modes
}

\author{
Nicolas TREPS \\ Laboratoire Kastler Brossel, Université Pierre et Marie Curie, Paris \\ nicolas.treps@upmc.fr
}

Du fait de ses potentielles applications, la mise en forme spatiale de la lumière est une des problématiques d'optique les plus actives. Ses succès sont déjà multiples, que ce soit en astronomie ou imagerie biologique, via l'optique adaptative, ou encore pour la focalisation à travers des milieux diffusants. L'optique quantique aussi y a puisé de l'inspiration, pour permettre en particulier d'ordonner spatialement les fluctuations quantiques et réaliser des expériences de métrologie quantique. De manière remarquable, les procédés développés pour ces études fondamentales ont des applications en télécommunications par fibre optique et ont permis de fonder la société CAllabs.

\section{Les fluctuations quantiques}

La nature quantique de la lumière se manifeste, lors de sa mesure par un détecteur très sensible, par la granularité du signal mesuré, directement reliée à la distribution aléatoire des photons à l'intérieur du faisceau lumineux. Ce bruit sur la mesure, souvent appelé bruit de grenaille, ou shot noise en anglais, limite la sensibilité de la mesure ou, de manière équivalente, la quantité d'information que l'on peut coder dans un faisceau lumineux. Cette limite d'origine fondamentale fait l'objet de très nombreuses recherches, regroupées sous le nom de métrologie quantique. Depuis maintenant une trentaine d'années les chercheurs savent produire des faisceaux lumineux dont les photons sont ordonnés dans le temps, aussi appelés états comprimés, permettant de réaliser des mesures avec une sensibilité accrue par rapport au shot noise. Ces états restent difficiles à générer et sortent doucement des laboratoires d'optique quantique pour des premières applications in situ, comme par exemple dans les interféromètres à ondes gravitationnelles.
Lorsque l'on ne s'intéresse plus aux fluctuations d'intensité totale d'un faisceau lumineux, mais à d'autres paramètres macroscopiques tels que son pointé ou sa dimension transverse, la situation se complique mais les fluctuations quantiques restent le facteur limitant. Ainsi, la question s'est posée de savoir avec quelle sensibilité il est possible de mesurer le pointé d'un faisceau laser. Cette question a des implications très directes puisque cette mesure de pointé est utilisée dans de nombreux appareils de haute précision, et des sensibilités sub-nanométriques pour un faisceau laser d'environ un millimètre de diamètre sont régulièrement atteintes. Le domaine de l'imagerie quantique a permis d'apporter des réponses précises à cette problématique et la clé en est l'analyse modale de la lumière.

\section{L'imagerie quantique}

Il est nécessaire ici, pour bien comprendre comment se propage la lumière, d'aborder le concept de modes transverses. Lors de sa propagation, un faisceau

Nicolas Treps, lauréat du prix Jean Jerphagnon en 2013, a co-fondé la société CAllabs suite à des recherches menées en imagerie quantique, pour proposer des multiplexeurs spatiaux pour les télécommunications et développer des solutions de mise en forme de la lumière pour d'autres domaines industriels comme le traitement des métaux ou les lasers très haute puissance. lumineux, tel un laser par exemple, a une forme transverse qui caractérise l'image formée par ce faisceau. Cette forme évolue avec la propagation de la lumière, en suivant les lois données par les équations de Maxwell. Mais il est toujours possible de décomposer tout faisceau lumineux en une somme de formes simples, de telle sorte que la lumière se propageant est la somme pondérée de ces modes. Un exemple bien connu de tels modes fondamentaux est l'ensemble de modes de Hermite-Gauss (voir figure 1), modes propres des cavités optiques. Un autre exemple est celui des polynômes de Zernike, très utilisés pour décomposer les aberrations d'un faisceau optique : on peut les quantifier en calculant le poids de chacun de ces modes dans la forme du faisceau. Les modes transverses peuvent donc être considérés sous deux points de vue équivalents : soit ils décomposent la forme d'une image, soit ils sont des canaux d'information. C'est ce dernier point de vue qui nous intéresse ici.

Ainsi, de manière générale, toute petite déformation d'un faisceau laser peut être représentée par des contributions de modes transverses spécifiques. Pour la problématique du positionnement d'un faisceau laser, on montre facilement (voir figure 2) qu'un petit déplacement 


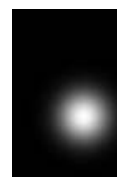

00

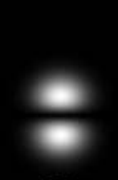

01

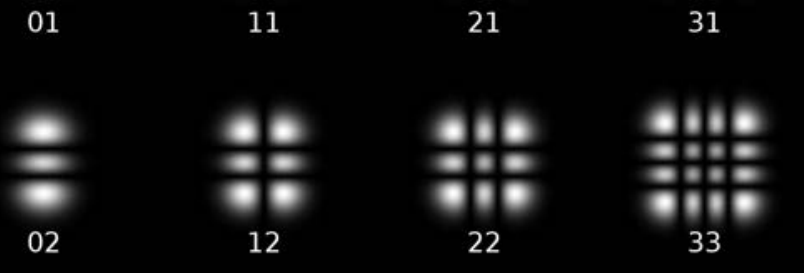

Ce principe est très général, et nous l'avons utilisé, en collaboration avec I'Université Nationale d'Australie, pour stabiliser le pointé d'un faisceau laser au-delà de ce qui est normalement imposé par les temps d'arrivée aléatoires des photons [1]. Pour cela nous avons généré un faisceau composite, dans lequel les fluctuations quantiques des modes

Figure 1. Forme transverse des premiers modes de Hermite-Gauss. Ces modes sont les modes propres des cavités optiques, et sont donc très bien adaptés à la décomposition de la forme d'un faisceau laser.

transverse du faisceau correspond, un peu comme avec une aberration du front d'onde optique, à l'ajout d'un mode transverse particulier. Réciproquement, la mesure de ce mode renseigne donc directement sur le positionnement relatif du faisceau par rapport au détecteur. Ce qui est remarquable, c'est que les fluctuations de pointé peuvent donc être exactement interprétées comme les fluctuations de champ électrique de ce mode, qu'elles soient d'origine classique ou quantique ! transverses responsables des fluctuations de pointé sont parfaitement contrôlées. Il a été nécessaire pour y parvenir de développer des techniques permettant de manipuler et de combiner les modes transverses - et ceci, sans perte.

\section{L'optique unitaire}

Les techniques nécessaires pour manipuler les modes transverses sont des techniques d'optique classique, même si elles ont été motivées par des problématiques quantiques. Il est donc possible de les présenter de manière assez générale. Le problème posé est le suivant: comment
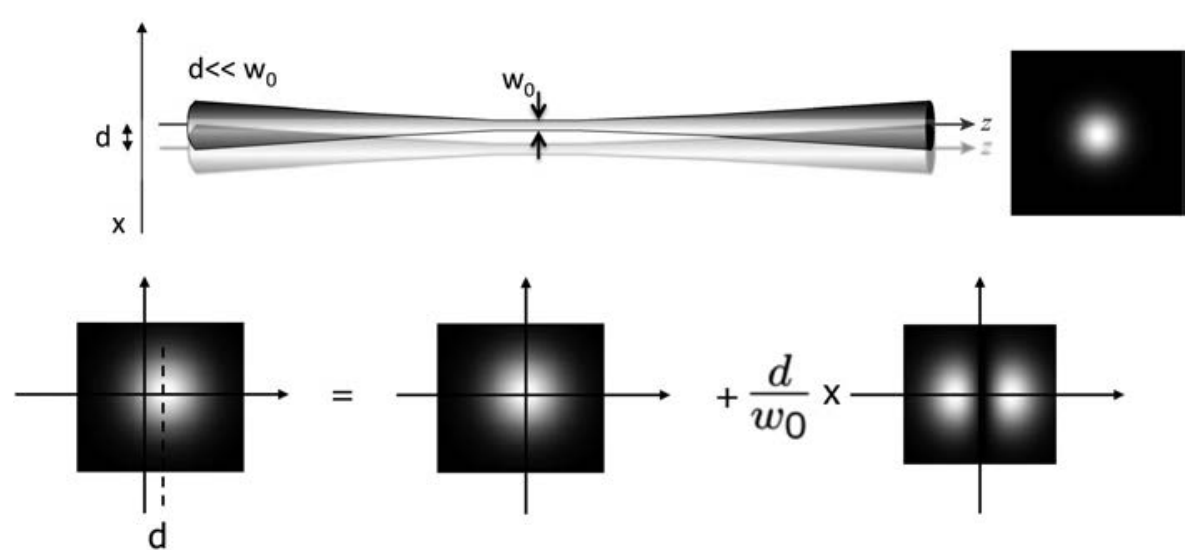

Figure 2. Si un faisceau gaussien est déplacé d'une petite quantité $d$ (exagérée sur la figure), au premier ordre ce déplacement est équivalent à l'ajout du premier mode de Hermite Gauss, avec wo le diamètre du faisceau au niveau du col. Ainsi, les fluctuations quantiques de ce mode induisent une limite quantique à la stabilisation du pointé d'un faisceau laser. Dans le cas des fluctuations du vide, on montre facilement que cette limite est en $1 / \sqrt{ } N$ où $N$ est le nombre de photons mesurés. Pour un faisceau $d^{\prime} 1 \mathrm{~mW}$ à $\lambda=1 \mu \mathrm{m}$, on a environ $10^{16}$ photons par seconde et donc une sensibilité de l'ordre de $10^{-8}$. En ordonnant les fluctuations quantiques du vide dans le mode de pointé, il est possible de stabiliser le faisceau au-delà de la limite quantique standard [1].

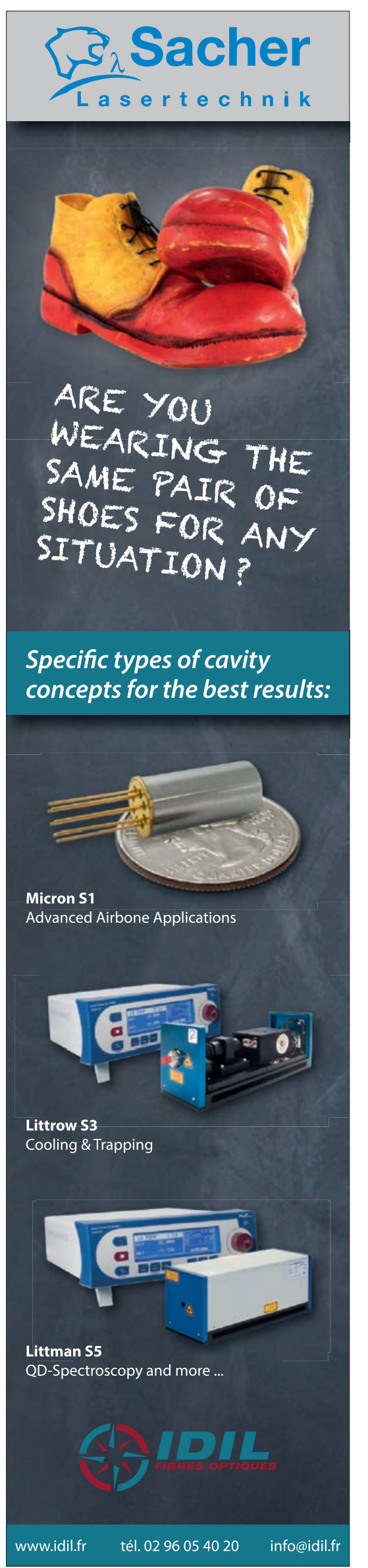

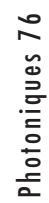




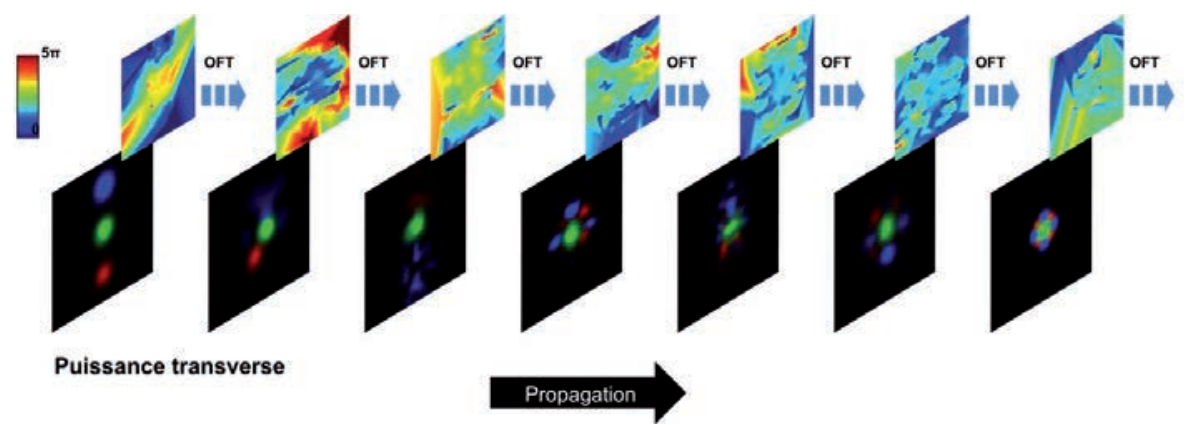

Figure 3. Exemple du mélange de trois faisceaux lumineux sans perte par transformations de phase successives. Les carrés noirs représentent des écrans successifs montrant la forme transverse des faisceaux lors de la propagation. À gauche, trois faisceaux lasers sont incidents et se propagent vers la droite. Au cours de leur propagation ils rencontrent des plans de phase (au-dessus) qui modifient la propagation et réalisent, au fur et à mesure, le mélange de ces trois faisceaux.

réaliser, sans perte, une transformation unitaire sur un faisceau lumineux? Unitaire signifiant ici que le faisceau incident et le faisceau de sortie sont chacun composés de $N$ modes, a priori différents, et que chaque mode d'entrée est dirigé vers un unique mode de sortie.

Le principe du système développé est inspiré de celui de l'optique adaptative. Dans ce dernier cas, un miroir déformable est placé sur le chemin d'un faisceau lumineux ayant subi des déformations de front d'onde, le plus souvent dues à des turbulences atmosphériques. Le miroir est alors adapté pour compenser localement les déformations de phase et retrouver toute la résolution optique d'un télescope par exemple. Ces systèmes très performants ont de nombreuses applications industrielles, mais ne permettent de traiter que des déformations de phase. Pour réaliser une transformation unitaire complète, permettant en pratique de modifier sans perte à la fois la phase et l'amplitude transverse d'un faisceau lumineux, il est nécessaire d'utiliser plusieurs plans de phase entre lesquels la lumière se propage. En choisissant judicieusement la distance entre les plans et en en utilisant suffisamment, on peut montrer que toute transformation sur la lumière peut être réalisée [2].

En figure 3 est donné un exemple où trois faisceaux lasers de même longueur d'onde (mais représentés sur la figure par trois disques de couleurs différentes pour les identifier) se propagent à travers un dispositif multi-plans. Celui-ci est réglé pour qu'à la sortie les trois faisceaux soient mélangés, chacun prenant alors une forme spécifique. Ces trois formes spécifiques sont superposées et se propagent ensemble, formant un unique faisceau de forme complexe. Sur la figure, au-dessus des faisceaux sont représentés les différents masques de phase appliqués à la lumière, masques qui sont séparés d'une distance telle que la lumière subit une transformation de Fourier optique lors de sa propagation (similaire à ce qu'elle subirait en allant d'un foyer optique à l'autre d'une lentille).

\section{Les télécommunications par fibre optique}

Ce système optique remarquable ouvre des possibilités d'applications très importantes, car il réalise une transformation qu'aucun autre système n'était jusqu'alors capable de réaliser. La première application développée l'a été en collaboration avec Alcatel Lucent et dans le cadre du projet européen SASER. L'objectif est d'utiliser les modes transverses pour augmenter le débit dans les fibres optique. En effet, les industriels des télécommunications s'interrogent sur l'augmentation constante des débits de données transportées et la capacité qu'auront les réseaux actuels pour les absorber. L'histoire des communications par fibre optique a été jalonnée de sauts technologiques (fibres monomodes, multiplexage spectral, etc.) qui ont permis d'augmenter de façon constante la capacité disponible dans les réseaux. Cependant depuis le début des années 2000 cette capacité stagne et aucune technologie n'est disponible. Le prochain saut technologique envisagé consiste à utiliser la forme de la lumière pour transporter de l'information dans des fibres optiques nouvelle génération, les few mode fibers comme illustré en figure 4.

Ces fibres, de taille pratiquement équivalente aux fibres actuelles, sont néanmoins capables de transporter jusqu'à une dizaine de modes transverses simultanément, augmentant d'autant la capacité des réseaux. Le système de transformation multi-plan décrit en figure 2 est le système qui manquait pour réaliser l'injection sans pertes d'une telle fibre.

\section{La sociéłé CAlLabs}

La société CAILabs (www.cailabs.com) a été fondée à partir des résultats de ce premier démonstrateur réalisé au laboratoire Kastler Brossel (LKB - Université Pierre et Marie Curie, École normale supérieure, CNRS). Bien entendu, l'une des toutes premières problématiques a été de savoir s'il pouvait être rendu compatible avec un environnement industriel. En effet, dans le cas du démonstrateur, la déformation de phase est réalisée avec un modulateur de phase spatial : il s'agit d'une matrice de cristaux liquides permettant de manière dynamique d'adresser la phase locale d'un faisceau. Mais ce système, coûteux, actif, encombrant, a dû être remplacé par une lame de phase fixe placée dans un système optique intégré (une cavité multi-passage) pour développer un système compact

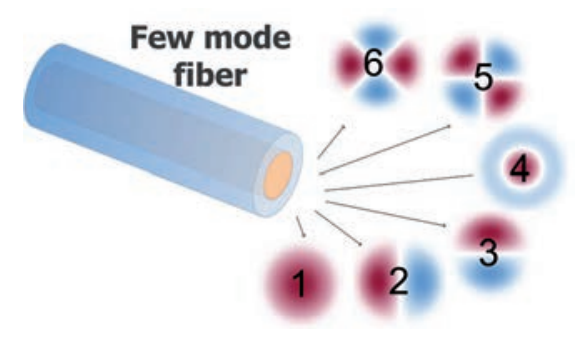

Figure 4. Fibre optique à petit nombre de modes ou few mode fiber. Sont représentées autour les formes transverses des faisceaux pouvant se propager simultanément et sans se mélanger dans la fibre. Une fibre à petit nombre de modes peut transporter jusqu'à dix modes transverses et donc dix fois plus d'information qu'une fibre standard. 


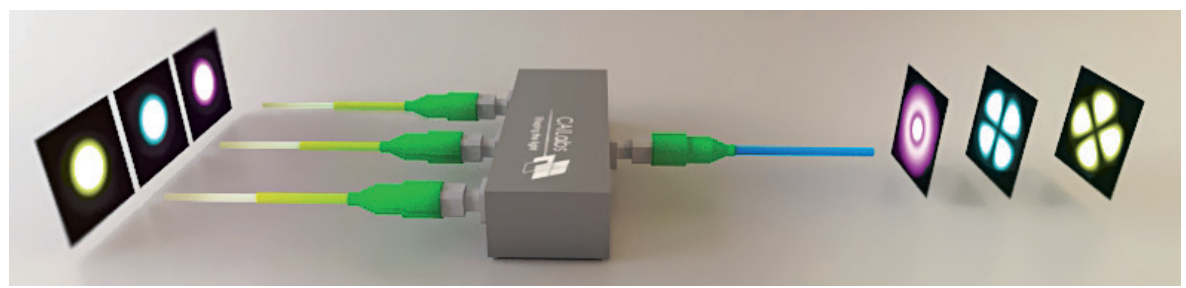

Figure 5. Multiplexage spatial pour le transport de l'information réalisé par le système CAllabs. Les images, en fausses couleurs, représentent les formes des différents modes à l'entrée et à la sortie du multiplexeur. Le démultiplexage s'effectue avec le même appareil utilisé dans l'autre sens.

permettant d'obtenir les premiers résultats de multiplexage et de demultiplexage spatial [3] (voir figure 5).

Depuis, les performances du système se sont très nettement améliorées et des multiplexeurs - démultiplexeurs à 6 modes sont disponibles commercialement. De plus, de nombreuses autres applications sont en cours de développement. On citera en particulier la mise en forme de faisceaux lasers de haute puissance, pour leur fonctionnalisation (à des opérations de soudure spécifique par exemple). En effet, une transformation unitaire permet à la fois d'éviter les pertes de puissance et de limiter la dissipation thermique, elle est donc extrêmement prometteuse pour toutes les applications où ces paramètres sont critiques.

\section{Et la suite?}

La société CAILabs est maintenant lancée et prospère indépendamment de ses organismes d'origine, et bien entendu de la physique quantique, dont elle est indirectement dérivée ! Néanmoins, les avancées apportées par le développement en milieu industriel du convertisseur multiplan permettent d'envisager son retour vers les laboratoires de recherche, car il ouvre de très nombreuses possibilités dans l'étude des milieux désordonnés et des systèmes optiques complexes par exemple. On peut donc raisonnablement penser que de nouvelles avancées sont à prévoir grâce à cet aller-retour constant entre des approches de physique fondamentale et de physique appliquée.

\section{Références}

[1] N. Treps, N. Grosse, W. Bowen, C. Fabre, H.A. Bachor, P. Lam, Science 301, 940 (2003).

[2] J.-F. Morizur, L. Nicholls, P. Jian, S. Armstrong, N. Treps, B. Hage, M.T.L. Hsu, W. Bowen, J. Janousek, H.-A. Bachor, JOSA a, 27, 2583 (2010)

[3] G. Labroille, B. Denolle, P. Jian, P. Genevaux, N. Treps, J.-F. Morizur, Opt Express 22 15599 (2014).

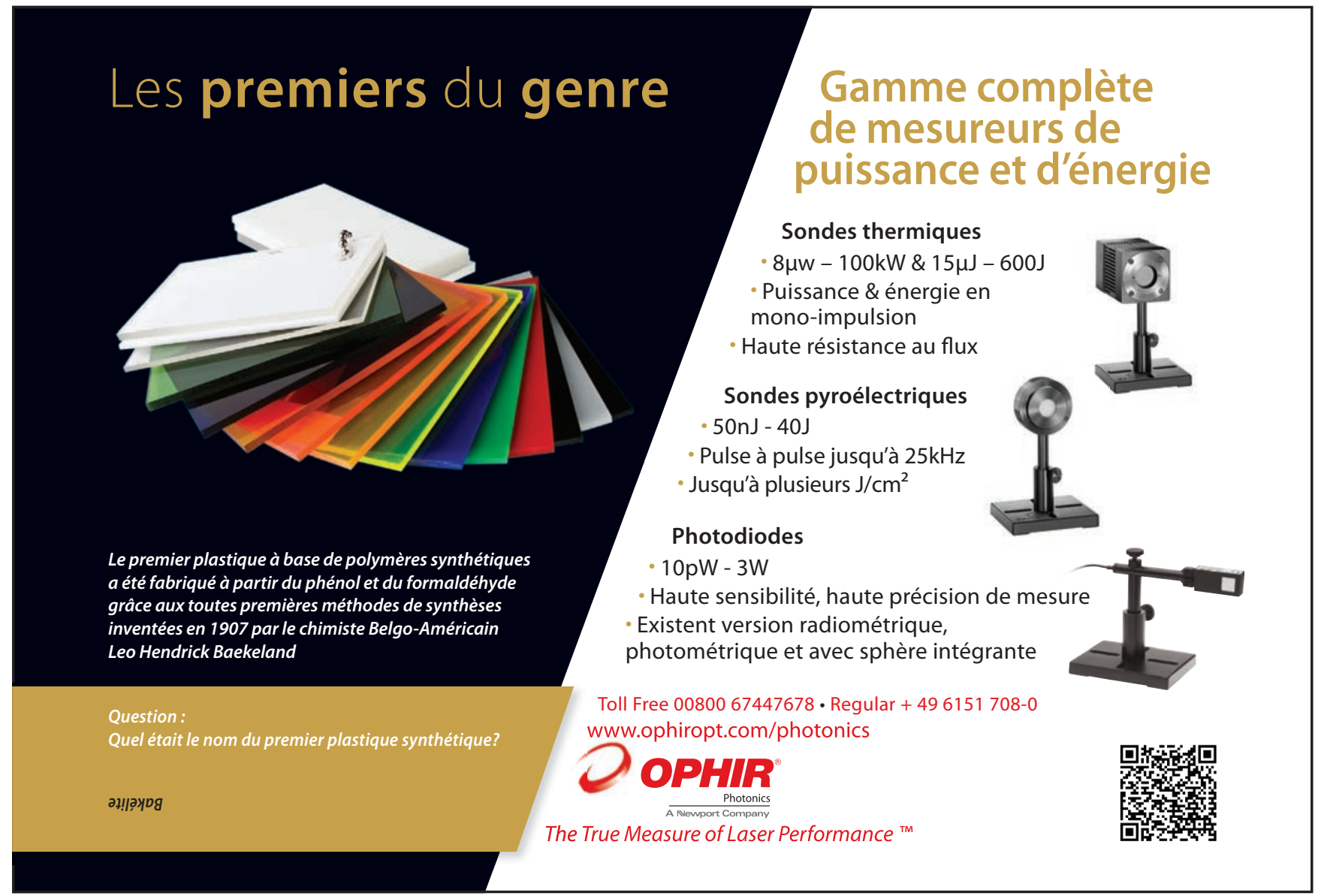

